\title{
View Factors for Surface Zones
}

\author{
R. P. Koptelov, G. K. Malikov, and V. G. Lisienko \\ Ural Federal University \\ Received July 31, 2012
}

\begin{abstract}
Methods of calculating view factors with different surface configurations are compared. A simple approach is proposed for estimating the error in calculating the view factors. An algorithm is formulated for selection of the method and the number of points of integration prior to the calculation. The algorithm significantly reduces the computation time by minimizing the number of integration points required, without loss of precision.
\end{abstract}

DOI: $10.3103 / \mathrm{S} 096709121301004 \mathrm{X}$

Detailed calculations of high-temperature energy systems and furnaces require precise models of radiant heat transfer [1, 2]. The simulation of radiant heat transfer necessitates the calculation of many view factors, including those for radiation between the boundary surfaces of grid. In some cases (electrical resistance furnaces, inductive heating, furnaces with radiation pipes and with a protective atmosphere, etc.), the medium between the surfaces may be neglected.

With no absorbing and scattering medium in the radiation path, the heat flux to surface $i$ may be written in the following form by the zonal method [1]

$$
Q_{i}=A_{i} \sum_{j=1}^{M} F_{i j} \int_{0}^{\infty}\left(J_{\lambda, i}-J_{\lambda, j}\right) d \lambda,
$$

where $F_{i j}$ is the view factor between diffuse surfaces $i$ and $j ; A$ is the surface area; $M$ is the number of surfaces participating in heat transfer; $J$ is the effective radiant heat flux; $J_{\lambda, i}$ is the spectral heat flux of the effective radiation; $\lambda$ is the radiant wavelength. The view factor $F_{i j}$ is the proportion of the radiant energy emitted and reflected by surface $i$ that travels directly to surface $j$ [3]. In metallurgical furnaces with a large number of zones, we may need to calculate tens (or even hundreds) of thousands of view factors $F_{i j}$ [4]. That is a particular concern when calculating the heat fluxes and temperatures in a large number of surface zones of the furnace.

The view factors may be calculated for each pair of surfaces in all the surface zones and written in matrix form. The matrix of view factors does not depend on the surface temperature. Therefore, it may be calculated once and then used in all subsequent steps to calculate the surface temperatures and heat fluxes. This is an important benefit of view factors, but the following problems must be noted.

(1) The calculation of the view factors for $M$ surfaces is associated with complexity of order $O\left(M^{2}\right)$.
Therefore, the algorithms that are satisfactory for a small number of surfaces may be too slow for a large number of surfaces [5].

(2) It is difficult to monitor the precision of the calculations. Two methods of monitoring the calculation precision, but both are heuristic. The first employs additive integration. The integral $I$ is calculated twice, with different number of integration points $n_{1}$ and $n_{2}$ $\left(n_{2}>n_{1}\right)$. If the difference between the results is small $\left|I\left(n_{1}\right)-I\left(n_{2}\right)\right|<\varepsilon$, then we take $I\left(n_{2}\right)$ as the value of the integral. Otherwise, we need to increase the number of integration points [5]. This approach permits a posteriori estimation of the error. The second approach applies to the Monte Carlo method. There is an approximate rule according to which the mean computational error for the view factors is inversely proportional to the square root of the number of beams emitted. However, this rule is only applicable to the mean error and not to the error in calculating a specific view factor [3].

\section{CALCULATING THE VIEW FACTORS}

First, we consider four methods of calculating the view factors from [5]. Then we briefly consider other options.

\section{Double Surface Integration}

In this case, the basic formula for the view factor $F_{12}$ involves a double surface integral

$$
F_{12}=\frac{1}{\pi A_{1}} \int_{A_{1} A_{2}} \int_{A^{2}} \frac{\cos \left(\theta_{1}\right) \cos \left(\theta_{2}\right)}{r^{2}} d A_{2} d A_{1},
$$

where $A_{1}$ and $A_{2}$ are the areas of surfaces 1 and $2 ; \theta_{1}$ and $\theta_{2}$ are the angles between the normals $\bar{n}_{1}$ and $\bar{n}_{2}$ and the centers of elementary areas $d A_{1}$ and $d A_{2} ; r$ is the distance between these elementary areas (Fig. 1). 


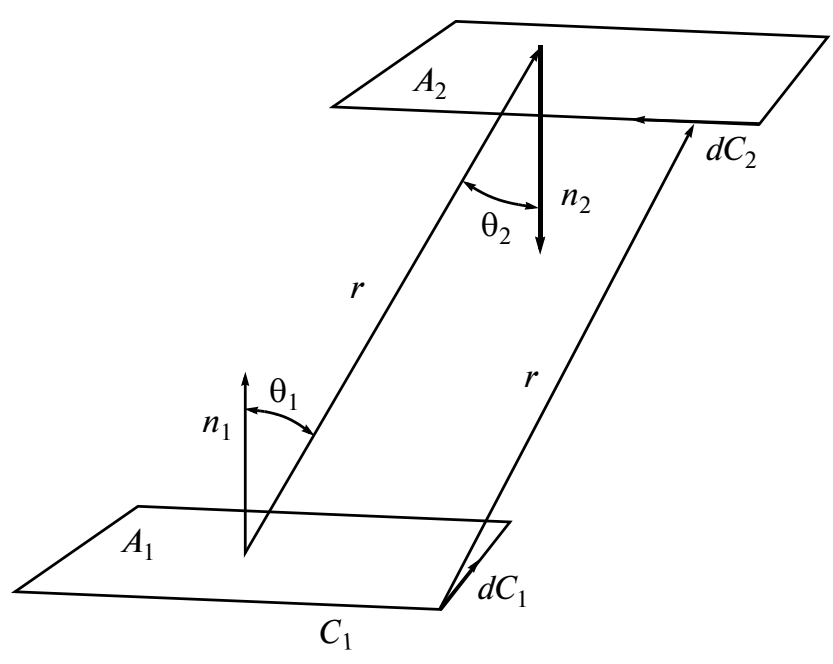

Fig. 1. Double surface integration and double contour integration.

\section{Double Contour Integration}

Using the Stokes theorem, the surface integrals in Eq. (2) are converted to contour integrals

$$
F_{12}=\frac{1}{\pi A_{1}} \oint_{C_{1} C_{2}} \oint_{C_{2}} \ln (r) d \bar{c}_{1} d \bar{c}_{2}
$$

where $C_{1}$ and $C_{2}$ are the boundary contours of the surfaces $A_{1}$ and $A_{2} ; d \bar{c}_{1}$ and $d \bar{c}_{2}$ are elementary segments of the corresponding contours; $r$ is the distance between the center of these elementary segments (Fig. 1).

\section{Single Surface Integration}

The view factor between an infinitesimal area and a finite area (a polygon) may be calculated from the formula [6]

$$
d F_{12}=\frac{1}{2 \pi} \sum_{j=1}^{N E_{2}} \bar{g}_{j} \bar{n}_{1},
$$

where $N E_{2}$ is the number of sides of the polygon; $\bar{g}_{j}$ is a vector whose length corresponds to the central angle with a chord equal to the length of a side of the polygon and with its center on the infinitesimal area. The vector $\bar{g}_{j}$ runs along the normal to the plane formed by the side of the polygon and a point on the area $d A_{1} ; \bar{n}_{1}$ is the normal to surface 1 . The formula is illustrated in Fig. 2a. Calculation by this formula is equivalent to the unit sphere method [1].

The view factor between two polygons may be calculated by the integration of Eq. (5)

$$
d F_{12}=\frac{1}{2 \pi A_{1}} \int_{A_{1} j=1}^{N E_{2}} \bar{g}_{j} \bar{n}_{1} d A_{1} .
$$

\section{Single Contour Integration}

In the case of a straight integration contour (side $E$ ), one of the integrals in Eq. (3) may be calculated analytically [7]

$$
\begin{aligned}
& I_{2}=\int_{\bar{E}} \ln (r) d \bar{E}=\left[r_{1} \ln r_{1} \cos \left(r_{1} \wedge E\right)\right. \\
& \left.+r_{2} \ln r_{2} \cos \left(r_{2} \wedge E\right)+h\left(r_{1} \wedge r_{2}\right)-|E|\right],
\end{aligned}
$$

(a)

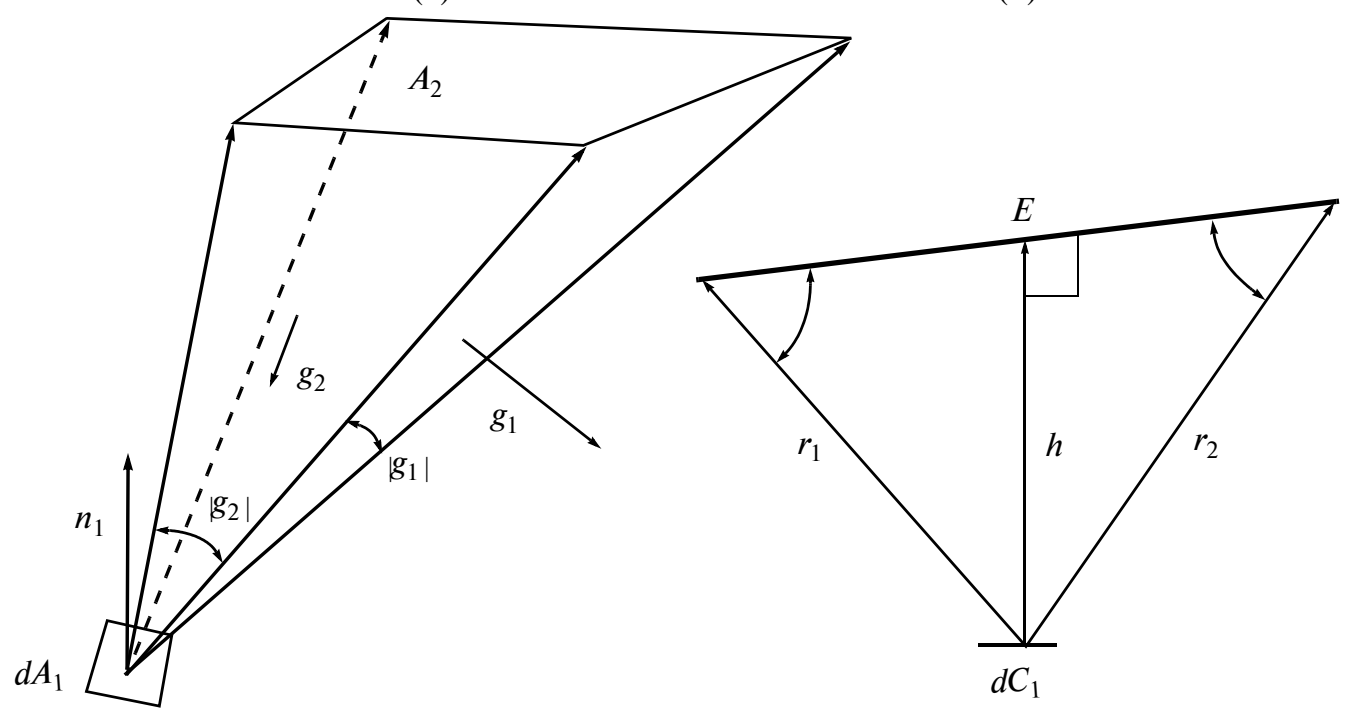

(b)

Fig. 2. Single surface integration (a) and single contour integration (b). 
where $|E|$ is the length of side $\bar{E} ; r_{1}$ and $r_{2}$ are the distances between the elementary contour segment $d \bar{c}_{1}$ and the ends of side $E ;\left(r_{1} \wedge r_{2}\right)$ is the angle between the vectors $\bar{r}_{1}$ and $\bar{r}_{2} ; h$ is the length of the perpendicular dropped from $d \bar{c}_{1}$ to side $E$ (Fig. 2b). Other versions of this formula may be found in $[3,5]$.

\section{Analytical Solution}

Analytical solution of the double contour integral was proposed in [8]. However, this solution is of little practical interest, on account of the computation time required. Such analytical solution is a hundred times slower than any of the four integration methods if we use four points for each integration variable, according to [5] (for example, if we use $16 \times 4^{2}$ points in the double contour integral and $4^{4}=256$ points in the double surface integral).

\section{Other Methods}

Examples of methods suited to calculating the view factors in complex systems with obstacles on the radiation path include the Monte Carlo method [1] and projection methods. In all projection methods, the irradiated surface area is projected onto a special surface: a hemisphere in the unit sphere method; half of a cube in [9]; a plane in [10]; or the faces of a tetrahedron in [11]. The resulting projection is divided into pixels, and the surface zone that receives the energy transmitted through each pixel is established. Methods using projection on a hemisphere were reviewed in [12].

Several methods applicable to particular geometry are also known. The inside sphere method permits calculation of the view factors between axisymmetric surfaces [1], while the extended-fiber method [1] and collapsed dimensions method (CDM) [13] may be used for two-dimensional geometry.

\section{GEOMETRIC CRITERION FOR A PRIORI ERROR ESTIMATION}

\section{Rationale for Integration Methods}

The following factors account for the use of integration methods, rather than analytical solution, in calculating the view factors.

(1) In practice, precision of $1-5 \%$ or even $10 \%$ is required. The precision of the heat fluxes and temperatures depends not only on the computational precision of the view factors but also on the radiative properties of the surfaces. If the radiation characteristics are known to 5\% precision, the precise calculation of view factors simply consumes extra time, without greatly increasing the precision of the temperatures.

(2) Approximate methods permit the calculation of the view factors with sufficient accuracy much more rapidly than in analytical solution.
(3) Approximate methods may sometimes be adapted for the calculation of nondiffuse view factors [14] and view factors in complex geometry with obstacles [5]. Analytical solution cannot be used in those important cases.

In the present work, one goal is a priori estimation of the error in calculating the view factors. This permits the selection of integration parameters for each surface configuration, prior to calculating the view factor.

\section{Relation between the Surface Configuration and the Computational Error}

The double contour integral may be the most popular means of calculating the view factors. Only the distance between the surfaces appears in Eq. (3). Therefore, this distance may be selected as the criterion in assessing the error of computation methods. The formulas for the double surface integral and the single contour integral also contain the distance between points of the surfaces. Another possible parameter for assessing the error is the mutual inclination of the surfaces. However, we have not established any relation between this inclination and the computational error.

The view factors for two coaxial unit squares may be calculated by means of a double contour integral (Fig. 3). We see that the relative error declines rapidly with increase in the distance between the squares (their planes). Even for close squares, the view factor may be obtained with high precision. That is consistent with the results in [5]. For polygons in parallel planes, clearly, the distance between them is the distance between their planes. However, for an arbitrary configuration of the polygons, the distance between them is an indefinable concept. Therefore, if the elementary view factor between the closest points is to be found with specified precision, the mean view factor must be found with even higher precision.

However, some problems arise here.

(1) The minimum distance must be calculated for each pair of polygons; in other words, $M^{2}$ values must be calculated.

(2) Calculation of the minimum distance between two polygons is a nontrivial problem. The minimum distance is the distance between the closest points However, the relative position of these points depends on the relative position of the polygons. These points may be at vertices of the polygons, at their sides, at an interior point, or in some combination (Fig. 4). Therefore, calculation of the minimum distance between polygons may take even more time than the calculation of the view factor if the number of integration points employed is small. 


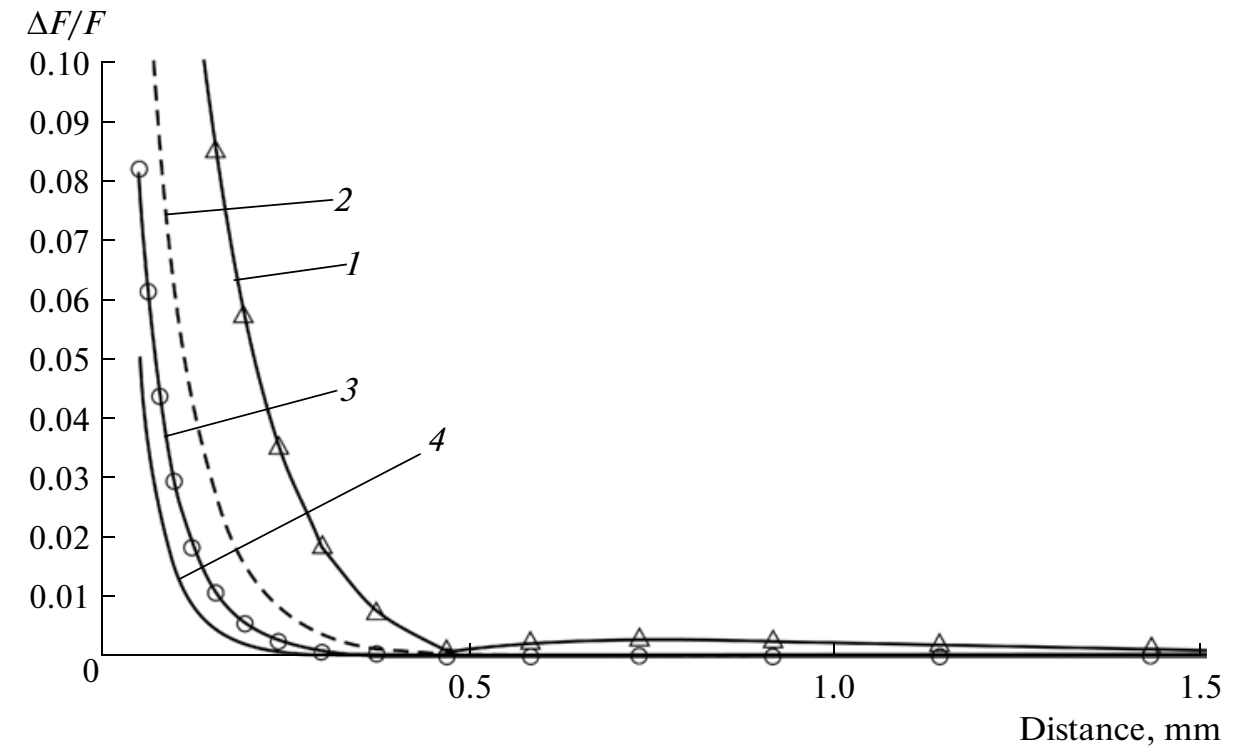

Fig. 3. Relative error in calculating the view factor for two unit coaxial squares when $n=2(1), 3$ (2), 4 (3), and 5 (4).

(a)

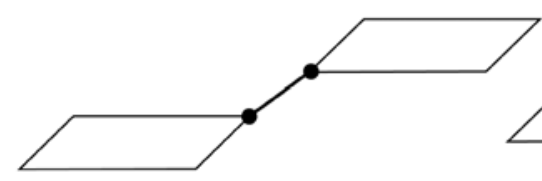

(b)

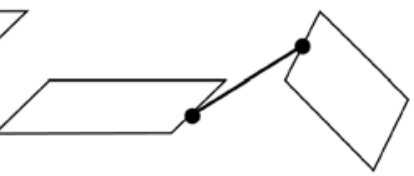

(c)

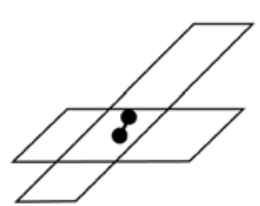

(d)

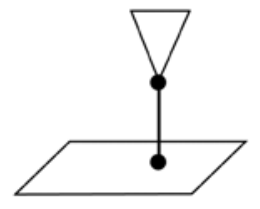

Fig. 4. Position of closest points on two polygons: (a) at vertices of the polygons; (b) at the sides; (c) at interior points; (d) from a vertex to an interior point.

\section{Effective Distance between Polygons}

We now consider an alternative to the minimum distance that is free of these deficiencies. Suppose that, for each polygon, we calculate the center and the radius of the bounding sphere. Any polygon may be enclosed within this sphere, but not all of its vertices will lie at its surface; in other words, this is not a circumscribing sphere. A simple algorithm for calculating the bounding sphere may be found in [15]. The sphere obtained is $5 \%$ larger, on average, than the minimum bounding sphere. The algorithm is executed in two passes. In the first pass, two very distant points (not necessarily the most distant points) are found. The initial sphere is constructed so that these are end points of its diameter. In the second pass, we verify whether particular points belong to the sphere. If not, the sphere is expanded appropriately.

We now introduce the effective distance between two polygons (or any other figures): the distance between the centers of the bounding spheres, divided by the sum of the radii of these spheres (Fig. 5) $\mathrm{ED}=$ $\left|O_{2}-O_{1}\right| /\left|R_{1}+R_{2}\right|$.

The effective distance offers the following benefits:
(1) the distance between the centers of the spheres characterizes the mean distance between the polygons;

(2) the effective distance is dimensionless;

(3) the calculation of the bounding sphere is simpler than the calculation of the minimum distance;

(4) the calculation of the bounding sphere is only necessary for each surface; that is, we need only calculate $M$ spheres, rather than $M^{2}$.

For each pair of surfaces, only calculation by the simplest formula is necessary ED $=\left|O_{2}-O_{1}\right| /\left|R_{1}+R_{2}\right|$.

\section{NUMERICAL EXPERIMENT}

The error in calculating the view factor depends on the distance between the planes for the case of two coaxial squares (Fig. 3). However, for practical use, we need a similar dependence for arbitrary tetragons, since in many cases tetragons are surface elements of a finite-difference grid. In the present work, the set of view factors is calculated for any configuration of the tetragons, and the relation between the effective distance and the computational precision is analyzed. 
(a)

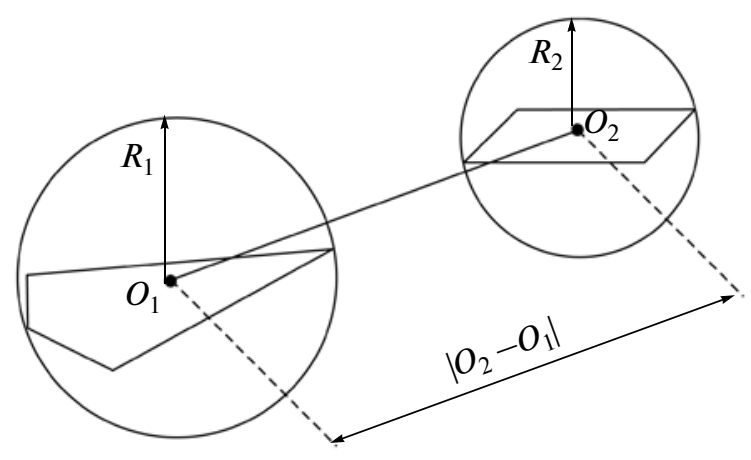

(b)

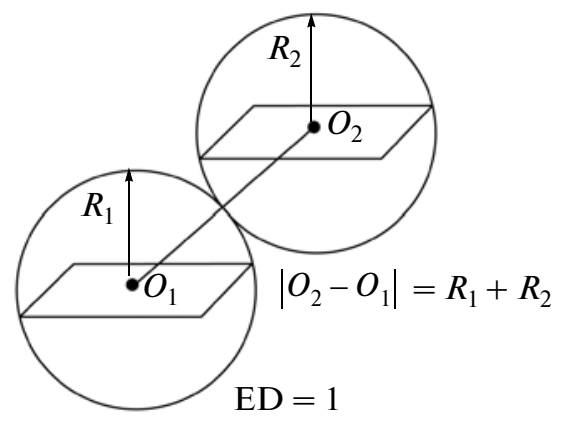

Fig. 5. Effective distance: (a) general case; (b) the particular case where $E D=1$ (contact of the spheres).

(a)

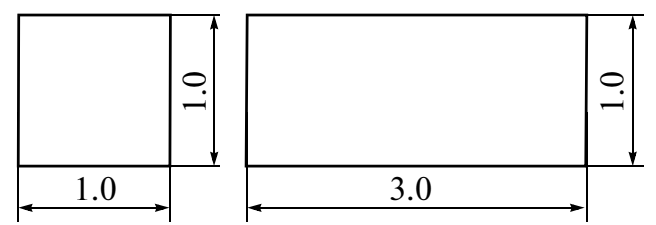

(c)

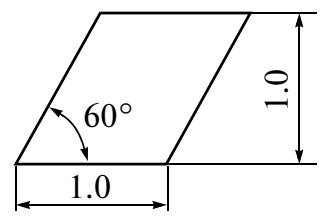

(d)

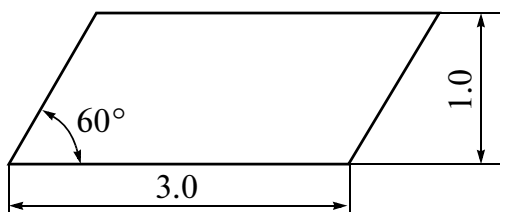

Fig. 6. Tetragons.

The tetragons are generated as follows. We use four basic tetragons (Fig. 6): a unit square (a), a rectangle (b); and two $60^{\circ}$ parallelograms with different side lengths (c, d). Such tetragons are often obtained when dividing a model of a furnace into zones.

The view factors are calculated for pairs of tetragons of the same form. The first tetragon is in the plane $z=0$ and is not subsequently transformed. Its area is always one, and its center is at the coordinate origin. The second tetragon is transformed to a different tetragon of the same shape but different size, by displacement relative to the coordinate origin and tilting (Fig. 7).
We use the following set of scale factors: $0.1,0.5$, $1.0,2.0$, and 10.0. The vertical displacement takes 18 values in a geometric progression with a 1.3 increment: $0.1,0.13, \ldots, 8.7$. The vertical displacement is always greater than zero. Therefore, the tetragons do not lie in a single plane. The horizontal displacement (along the $x$ axis) corresponds to the same progression, except that the first value is zero. The tetragon is rotated around its $x$ and $z$ axes, within the range from 0 to $\pi / 2$ (increment $\pi / 18$ ). The scale factor, vertical and horizontal displacements, and angles of rotation vary independently. The total number of different configurations of the tetragons is $5 \times 10^{5}$. (a)

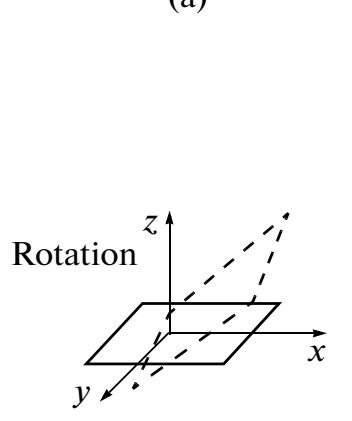

(b)

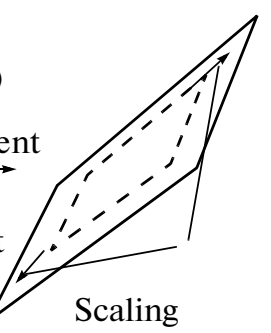

(c)

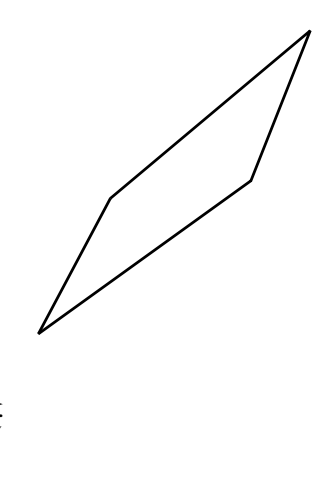

Fig. 7. Transformation of the second polygon: (a) rotation; (b) displacement and scaling; (c) final position after transformation. 


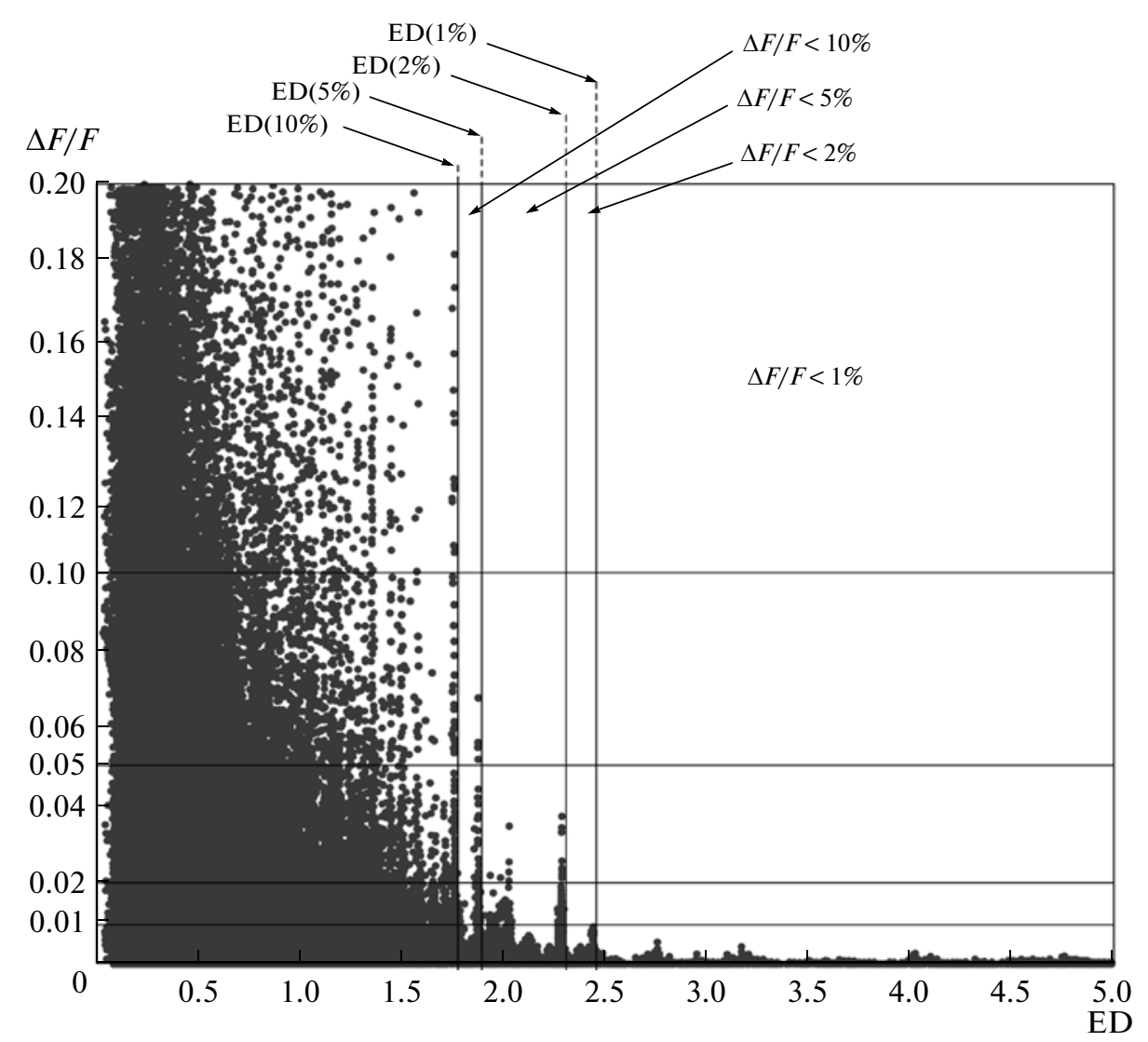

Fig. 8. Relation between the relative error in calculating the view factor and the effective distance. Each point represents one of $5 \times 10^{5}$ view factors. Only those larger than $10^{-4}$ are analyzed.

\section{RESULTS}

The view factors for the set of tetragons are calculated by means of four methods: double surface integration; single surface integration; double contour integration; and single contour integration. Quadratic Gaussian formulas (the product of onedimensional formulas) are used throughout. In the formulas, $n$ is the number of integration points for a single integration variable. In other words, the total number of integration points $N$ is $16 n$ in the single contour integral; $n^{2}$ in the single surface integral; $16 n^{2}$ in the double contour integral; and $n^{4}$ in the double surface integral.

In Fig. 8, we show the attainable relative error when using a double contour integral with $n=3(N=$ $16 \times 9=144)$.

We see that the error declines with increase in the effective distance. We may find values $\operatorname{ED}(10 \%)$, $\mathrm{ED}(5 \%), \mathrm{ED}(2 \%)$, and $\mathrm{ED}(1 \%)$ such that, if $\mathrm{ED}_{i j} \mathrm{cal}-$ culated for tetragons $i$ and $j$ is greater than $\operatorname{ED}(5 \%)$, say, then the error may be estimated a priori as less than $5 \%$, since that is the case for all possible configurations of the tetragons (Fig. 8). In other words, we establish the following relationships

$$
\begin{aligned}
\mathrm{ED}_{i j}<\mathrm{ED}(10 \%) & \rightarrow \Delta F_{i j} / F_{i j}<10 \%, \\
\mathrm{ED}_{i j}<\mathrm{ED}(5 \%) & \rightarrow \Delta F_{i j} / F_{i j}<5 \%, \\
\mathrm{ED}_{i j}<\mathrm{ED}(2 \%) & \rightarrow \Delta F_{i j} / F_{i j}<2 \%, \\
\mathrm{ED}_{i j}<\mathrm{ED}(1 \%) & \rightarrow \Delta F_{i j} / F_{i j}<1 \%,
\end{aligned}
$$

and so on for any precision.

We now determine $\operatorname{ED}(10 \%), \operatorname{ED}(5 \%), \operatorname{ED}(2 \%)$, and $\operatorname{ED}(1 \%)$ with different numbers of integration points by the four methods (Table 1). In the first three methods, we use the elementary precision in the calculations ( 4 bytes). However, that is insufficient when using a single contour integral. When the same accuracy is used in the single contour integral, an error less than $10 \%$ cannot be obtained even with a large number of integration points. Therefore, in that case, we use double precision.

The values obtained by single contour integration with $n=40 \mathrm{~s}$ at double precision are regarded as precise values of the view factors. Fortran is used for all the calculations, on a $1.83-\mathrm{GHz}$ Pentium processor. 
Table 1. ED values for calculating the view factor with specified precision

\begin{tabular}{|c|c|c|c|c|c|c|c|}
\hline $\begin{array}{l}\text { Computation } \\
\text { method }\end{array}$ & $\begin{array}{c}n \text {, number of points } \\
\text { in one-dimensional } \\
\text { interval }\end{array}$ & $\begin{array}{l}N \text {, total number } \\
\text { of integration } \\
\text { points }\end{array}$ & $\begin{array}{l}\text { Computational } \\
\text { time for } 10^{6} \text { view } \\
\text { factors, } \mathrm{s}\end{array}$ & $\mathrm{ED}(10 \%)$ & $\operatorname{ED}(5 \%)$ & $\operatorname{ED}(2 \%)$ & $\mathrm{ED}(1 \%)$ \\
\hline \multirow{6}{*}{$\begin{array}{l}\text { Double surface } \\
\text { integral }\end{array}$} & 1 & $1^{4}=1$ & 0.66 & 4.10 & 6.20 & 7.40 & 9.20 \\
\hline & 2 & $2^{4}=16$ & 2.04 & 1.55 & 2.00 & 2.60 & 2.95 \\
\hline & 3 & $3^{4}=81$ & 5.92 & 1.20 & 1.35 & 1.55 & 1.60 \\
\hline & 4 & $4^{4}=256$ & 15.02 & 1.05 & 1.20 & 1.20 & 1.35 \\
\hline & 5 & $5^{4}=625$ & 32.76 & 0.9 & 1.05 & 1.05 & 1.20 \\
\hline & 6 & $6^{4}=1296$ & 64.14 & 0.80 & 0.90 & 1.00 & 1.05 \\
\hline \multirow{6}{*}{$\begin{array}{l}\text { Single surface } \\
\text { integral }\end{array}$} & 1 & $1^{2}=1$ & 1.86 & 3.60 & 5.10 & 8.40 & 10.20 \\
\hline & 2 & $2^{2}=4$ & 6.89 & 1.55 & 2.00 & 2.60 & 2.95 \\
\hline & 3 & $3^{2}=9$ & 15.13 & 1.20 & 1.35 & 1.55 & 1.60 \\
\hline & 4 & $4^{2}=16$ & 26.65 & 1.05 & 1.20 & 1.20 & 1.35 \\
\hline & 5 & $5^{2}=25$ & 41.51 & 0.90 & 1.05 & 1.05 & 1.20 \\
\hline & 6 & $6^{2}=36$ & 59.74 & 0.80 & 0.90 & 1.00 & 1.05 \\
\hline \multirow{6}{*}{$\begin{array}{l}\text { Double con- } \\
\text { tour integral }\end{array}$} & 1 & $16 \times 1^{2}=16$ & 3.33 & 6.30 & 6.65 & 8.70 & 9.10 \\
\hline & 2 & $16 \times 2^{2}=64$ & 7.41 & 2.45 & 3.20 & 3.20 & 3.70 \\
\hline & 3 & $16 \times 3^{2}=144$ & 13.75 & 1.80 & 1.90 & 2.30 & 2.30 \\
\hline & 4 & $16 \times 4^{2}=256$ & 22.37 & 1.35 & 1.45 & 1.45 & 1.80 \\
\hline & 5 & $16 \times 5^{2}=400$ & 33.35 & 1.05 & 1.15 & 1.35 & 1.35 \\
\hline & 6 & $16 \times 6^{2}=576$ & 46.69 & 1.05 & 1.05 & 1.05 & 1.15 \\
\hline \multirow{4}{*}{$\begin{array}{l}\text { Single contour } \\
\text { integral }\end{array}$} & 1 & $16 \times 1=16$ & 10.22 & 6.25 & 6.65 & 8.70 & 9.10 \\
\hline & 2 & $16 \times 2=32$ & 18.07 & 2.25 & 2.25 & 2.95 & 3.35 \\
\hline & 3 & $16 \times 3=48$ & 25.86 & 1.50 & 1.50 & 1.75 & 2.00 \\
\hline & 4 & $16 \times 4=64$ & 33.59 & 0.90 & 1.05 & 1.15 & 1.35 \\
\hline
\end{tabular}

Using Eqs. (7)-(10) and the data in Table 1, we may formulate an algorithm for selecting the method of integration and the number of points.

(1) For all the view factors, we select the desired accuracy $(x \%)$. That means that we select the corresponding column in Table 1.

(2) For all the surfaces, we calculate the bounding sphere.

(3) For each pair of tetragons $i, j$, we calculate $\mathrm{ED}_{i j}$. For each method, we find the minimum $n$ such that $\mathrm{ED}(x \%, n) \leq \mathrm{ED}_{i j}<\mathrm{ED}(x \%, n+1)$.

The left side of this inequality permits satisfaction of the precision requirement, while the right side ensures the use of the minimum number of points.

(4) On the basis of the values of $n$ for each of the four methods and the corresponding computation time, we may select the fastest method for calculating $F_{i j}$.

It is evident from Table 1 that the precision strongly depends on the selected effective distance, while the view factors may generally be calculated using a small number of integration points. We may use ED for most possible surface configurations. However, a defect of the method is apparent here. To meet the precision requirement in all possible cases, the ED value is overestimated. For particular cases, ED may be much lower. For example, the radii of the bounding spheres for two coaxial squares are $R_{1}=R_{2}=1 / \sqrt{2}$. If the squares are at unit distance, then $\mathrm{ED}=\sqrt{2} / 2 \approx 0.71$. In that case, $10 \%$ precision cannot be attained, to judge from Table 1. However, we see in Fig. 3 that, in fact, even $n=2$ is sufficient to obtain $1 \%$ precision. That means that the minimum number of points obtained from Table 1 with ED $>1$ cannot be determined with $\mathrm{ED}<1$, especially if $1 \%$ precision is required.

We now determine the proportion of the view factors for which the sufficient number of points may be calculated using Table 1. If ED > 1 for two tetragons, the view factor may be calculated by any of the methods, with a small number of points. Note that ED $=1$ if the bounding spheres of the tetragons touch (Fig. 5b). Hence, ED > 1 if the bounding spheres do not intersect and do not touch. Then the proposed algorithm may be used for all pairs of tetragons whose bounding spheres do not touch. The proportion of such tetragonal surfaces may be estimated on the basis 
Table 2. Proportion of the view factors that may (MC) and may not (MNC) be calculated by the proposed algorithm

\begin{tabular}{l|c|c|c|c|c|c|c}
\hline $\begin{array}{l}\text { Number } \\
\text { of surfaces } \\
(M-9) /(M-1),\end{array}$ & 30 & 50 & 100 & 150 & 200 & 400 & 1000 \\
$\begin{array}{l}\%(\mathrm{MC}) \\
\begin{array}{l}8 /(M-1), \% \\
(\mathrm{MNC})\end{array}\end{array}$ & 27.6 & 16.3 & 8.1 & 5.4 & 4.0 & 2.0 & 0.8 \\
\hline
\end{tabular}

of the following simplification: the bounding spheres of two surfaces intersect only of the surfaces are adjacent - that is, if they have a common side. For each of the $M$ surfaces, only eight are adjacent. For each surface, we need to calculate $M-1$ view factors: eight with the eight adjacent surfaces; and $(M-1)-8=$ $M-9$ with the other surfaces for which ED $>1$. Thus, $(M-9) /(M-1)$ view factors may be effectively calculated by the proposed algorithm; a different algorithm is needed for only $8 /(M-1)$ view factors. The corresponding proportions of view factors are presented in Table 2. We see that the proposed algorithm works for more than $90 \%$ of the view factors if the model consists of 100 surfaces and more than $99 \%$ if the model consists of 1000 surfaces. The adjacent surfaces constitute a small proportion of all the pairs of surfaces, and only for those do we need to resort to high-order quadrature formulas or analytic solution.

\section{CONCLUSIONS}

Numerical comparison of four methods of calculating the view factors between tetragonal surfaces has been undertaken for various configurations. For each method and for each fixed number of integration points, we have calculated more than $2 \times 10^{5}$ view factors.

The results show that the precision in calculating the view factor may be assessed a priori by means of a simple parameter: the effective distance. That permits a priori selection of the minimum number of integration points for each pair of tetragons. As a result, the time required to calculate the matrix of view factors may be considerably reduced, without loss of precision.

The proposed algorithm for selection of the number of integration points performs well when $E D \geq 1$. If $\mathrm{ED}<1$ (areas with a common side), integration with a much larger number of points is required, but expe- rience shows that, in most metallurgical applications, $\mathrm{ED}<1$ for only $1-10 \%$ of the total number of pairs of emitting areas.

\section{REFERENCES}

1. Modest, M.F., Radiative Heat Transfer, New York: Academic, 2003.

2. Lisienko, V.G., Sovershenstvovanie i povyshenie effektivnosti energotekhnologii $i$ proizvodstv. Integrirovanyi energo-ekologicheskii analiz: teoriya i praktika (Improving Electrical Technology in Production: Theory and Practice of Energy-Ecological Analysis), Moscow: Teplotekhnik, 2010, vol. 1.

3. Emery, A.F., Johansson, O., Lobo, M., and Abrous, A., ASME J. Heat Trans., 1991, no. 113, pp. 413-422.

4. Malikov, G.K., Lisienko, V.G., and Koptelov, R.P., Izv. Vyssh. Uchebn. Zaved., Chern. Metall., 2010, no. 7, pp. 53-59.

5. Walton, G.N., Calculation of Obstructed View Factors by Adaptive Integration, Washington: National Institute of Standards and Technology, 2002, NISTIR 6925.

6. Hottel, H.C. and Sarofim, A.F., Radiative Transfer, New York: McGraw-Hill, 1967.

7. Mitalas, G.P. and Stephenson, D.G., Fortran IV Program to Calculate Radiant Interchange Factors, Ottawa, Canada: NRC, 1966, DBR-25.

8. Schroder, P. and Hanrahan, P., A Closed Form Expression for the Form Factor between Two Polygons, Department of Computer Science, Princeton University, 1993, Technical Report CS-404-93.

9. Cohen, M.F. and Greenberg, D.P., ACM SIGGRAPH, 1985, no. 19(3), pp. 31-40.

10. Recker, R.J., George, D.W., and Greensberg, D.P., Computer Graphics, 1990, no. 24(2), pp. 59-66.

11. Beran-Koehn, J.C. and Pavicic, M.J., Delta Form Factor Calculations for the Cubic Tetrahedral Algorithm, Graphics Gems III, New York: Academic, 1992, pp. 324-328.

12. Kim, S. and Yoon, K., An Alternative to the Hemicube Algorithm for Computing Form Factors, Graphicon International Conference, Moscow, 2000.

13. Subhash, C.M., Shukla, A., and Yadav, V., Int. Comm. Heat Mass Trans., 2008, no. 35(5), pp. 630-636.

14. DiLaura, D.L., Proceedings of Thirty-Third National Heat Transfer Conference, Albuquerque, 1999, pp. 99107.

15. Glassner, A.S., Graphics Gems I, New York: Academic, 1990, pp. 301-303. 